PROFESIONALES Y HERRAMIENTAS PARA EL DESARROLLO LOCAL Y SUS SINERGIAS TERRITORIALES. EVALUACIÓN Y PROPUESTAS DE FUTURO IX Coloquio Nacional de Desarrollo Local del GTDL-AGE 

ANTONIO MARTÍNEZ PUCHE, XAVIER AMAT MONTESINOS, ISABEL SANCHO CARBONELL y DANIEL SANCHIZ CASTAÑO (EDS.)

\section{PROFESIONALES Y HERRAMIENTAS PARA EL DESARROLLO LOCAL Y SUS SINERGIAS TERRITORIALES. EVALUACIÓN Y PROPUESTAS DE FUTURO}

IX Coloquio Nacional de Desarrollo Local del GTDL-AGE

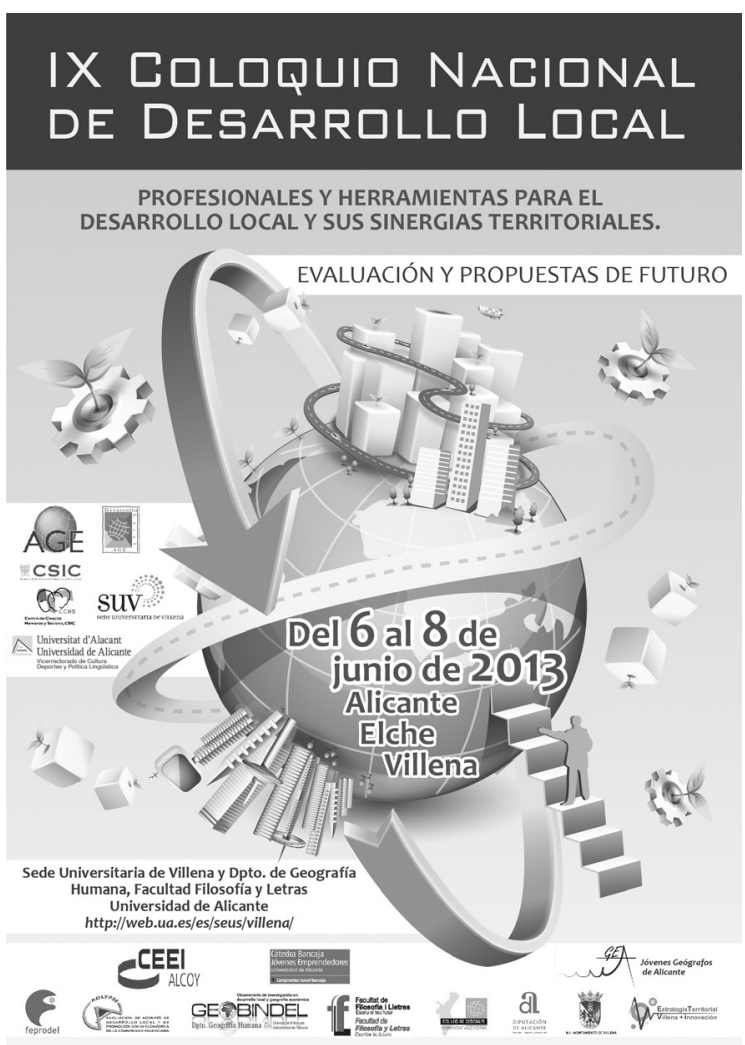


Este libro ha sido examinado y valorado por evaluadores ajenos a la Universidad de Alicante, con el fin de garantizar la calidad científica del mismo.

Publicacions de la Universitat d'Alacant

03690 Sant Vicent del Raspeig

Publicaciones@ua.es

http://publicaciones.ua.es

Telèfon: 965903480

(C) Antonio Martínez Puche, Xavier Amat Montesinos,

Isabel Sancho Carbonell y Daniel Sanchiz Castaño (eds.), 2016

(C) d'aquesta edició: Universitat d'Alacant

ISBN: 978-84-16724-00-0

Dipòsit legal: A 92-2016

Disseny de coberta: candela ink

Composició: Página Maestra (Miguel Ángel Sánchez Hernández)

Impressió i enquadernació: Guada Impresores

\section{unte \\ Unión de Editoriales
Universitarias Españolas \\ WWW.une.es
WWA}

Esta editorial es miembro de la UNE, cosa que garantiza la difusión y comercialización nacional y internacional de sus publicaciones.

Reservados todos los derechos. Cualquier forma de reproducción, distribución, comunicación pública o transformación de esta obra sólo puede ser realizada con la autorización de sus titulares, salvo excepción prevista por la ley. Diríjase a CEDRO (Centro Español de Derechos Repográficos, www.cedro.org) si necesita fotocopias o escanear algún fragmento de esta obra. 


\title{
DESARROLLO LOCAL SOSTENIBLE, CRISIS E INICIATIVAS LOCALES DE RESILIENCIA
}

\author{
Pere J. Brunet Estarellas \\ Departament de Ciències de la Terra, Universitat de les Illes Balears (UIB) \\ pere.brunet@uib.es
}

RESUMEN

El desarrollo local tiene como objetivo la mejora general de la población, que se pretende alcanzar de acuerdo con la voluntad consensuada y responsable de los diferentes agentes sociales, políticos y económicos que comparte un territorio concreto, a partir de la previa identificación y aprovechamiento de los recursos y potencialidades endógenas. Desde la difusión del término sostenible, a principios de la década de los 90 del siglo pasado, se ha ido consolidando el concepto de desarrollo local sostenible, mediante el que se ha promovido la idea de que el progreso local, además de económicamente viable, sea equitativo desde el punto de vista social y respetuoso desde la perspectiva ambiental.

En el caso español, y muy concretamente en las Islas Baleares, el análisis en profundidad de las estrategias seguidas para conseguir un desarrollo sostenible a escala local permite detectar éxitos y fracasos, así como las importantes dificultades con las que ha topado la propuesta a la hora de ser interpretada, adoptada y mantenida.

Y si en tiempo de bonanza, las razonables dudas de la clase política y el oscilante grado de implicación de los ciudadanos han hecho que iniciativas como la Agenda 21 Local (A21L) hayan tenido diferente nivel de implantación y permanencia, el distanciamiento entre los poderes públicos y la ciudadanía ha puesto a prueba la capacidad de los actores locales para activar los mecanismo tradicionales de desarrollo local frente a la crisis y adoptar nuevas fórmulas de organización colectiva y de resiliencia.

Palabras clave: Desarrollo local sostenible, Agenda 21 (A21L), Agentes de Empleo y Desarrollo Local (AEDL), Resiliencia, Islas Baleares. 


\section{LOCAL SUSTAINABLE DEVELOPMENT CRISIS AND LOCAL INITIATIVES OF RESILIENCE}

\section{AbSTRACT}

Local sustainable development, crisis and local initiatives of resilience.

Local development has the goal $\mathrm{f}$ improving overall population by the will consensual and responsable of the various social, political and economic actors that share a specific territory. Fot that it si necessary the identification and exploitation of endogenous resources and potentialities.

Since the diffusion of sustentable term in the early 90 s of the last century, the concept of sustainable local development has been conslidated. This concept promoted the idea that local progress, as wel as economically viable, has to be equitable from the standpoint of social and friendly with the environmental perspective.

In the Spanish case, specifically in the Balearic Islands, the in-depth analysis of the strategies for achieving sustainable development at local level, allows to detect successes and failures, as well as the significant difficulties that the proposal has encountered at the momento of being interpreted, adopted and maintained.

If iniciatives such as Local Agenda 21 (A21L) have had different levels of implementation and permanence depending on the political class and the participation level of citizens, the current distance between government and citizens has tested the ability of local actors to activate traditional local development mechanisms and adopt new forms of collective organization and resiliencie.

Key words: Sustainable Local Development, Local Agenda 21, Employment Agents and Local Development, Resilience, Balearic Islands.

\section{Desarkollo local y la CRISIS eCONÓMiCA}

El desarrollo local se concibe como el resultado de la voluntad de actores sociales, políticos y económicos de intervenir activamente en los procesos de desarrollo en sus respectivos territorios desde una perspectiva amplia e integrada (KLEIN, 2006). Con el tiempo, las acciones relacionadas con el desarrollo local se han convertido en una de las estrategias de crecimiento endógeno más importantes, especialmente en el marco de la inserción socio-laboral (SANCHIS, 1999).

Se trata de un modelo de intervención mediante la que, desde los años 70, se ha intentado responder y dar respuesta a los diferentes ciclos de crisis, especialmente en los momentos de incesante destrucción de puestos de trabajo, y que ha madurado y mejora a lo largo de las etapas de aparente bonanza y de convulsión siguientes. 
En el análisis del desarrollo local y, en particular, del papel jugado por los actores implicados los agentes de empleo y desarrollo local (AEDL) ha sido el colectivo al que posiblemente se ha prestado más atención. En cambio, son pocos los estudios que se hayan analizado en profundidad en los condicionantes impuestas por los poderes públicos locales en el desarrollo de sus actividades.

La necesaria relación entre técnicos y políticos en el proceso, hace conveniente identificar dos modelos de implantación y gestión de desarrollo local por parte de los municipios:

1. El llevado a cabo con recursos propios, con el objetivo de implementar acciones que sólo afectan a un municipio concreto.

2. El desplegado de acuerdo con los compromisos de participación con otros socios y administraciones en proyectos europeos o de otra naturaleza.

La diferencia entre estas dos alternativas no es menor. La información disponible al respecto permite relacionar los modelos descritos con aspectos tan relevantes como la permanencia o caducidad, el carácter estratégico o eventual, etc.

En la práctica, dichos modelos no suelen presentarse de forma tan rígida y adoptan una serie de características singulares para acomodarse mejor a las circunstancias del territorio.

En cualquier caso, en función de los diferentes criterios políticos y coyunturas, los poderes públicos deberán apostar por una metodología de desarrollo local determinado o renunciar a ello, o lo que es lo mismo:

1. Asumir los riesgos económicos y políticos derivados de la implantación y mantenimiento de un modelo de desarrollo local determinado que requiere dedicación e inversión.

2. Renunciar a principios y objetivos propios del desarrollo local, no adoptar o abandonar programas y acciones relacionadas con esta alternativa, por falta de liquidez o por considerar que las acciones implementadas para mejorar las condiciones de vida de la población no han generado beneficios substanciales.

Como sea que a lo largo de este trabajo haremos referencia explícita al caso balear, creemos oportuno decir que, como respuesta a la actual crisis, desafortunadamente, el Gobierno Balear y la mayor parte de municipios del archipiélago han adoptado en los últimos años el segundo tipo de medidas.

Dicha solución, precipitada y de corto alcance, sin duda no difiere a las que han sido adoptadas en otras Comunidades Autónomas. De hecho, se puede constatar que otros poderes públicos autonómicos y locales han aprobado similares políticas, basándose de forma casi exclusiva en criterios de ahorro y reducción de las inversiones públicas, en franca contradicción con el espíritu del desarrollo local y la necesidad de apostar por estrategias activas de empleo. Son decisiones alineadas con la práctica de una política dirigista y desprovista 
de imaginación suficiente para responder de manera eficiente a los retos de la actual crisis y que ha supuesto, entre otras cosas:

- Una clara ruptura del proceso de generación o reforzamiento de la dinámica de desarrollo local

- La pérdida de la visión transversal y tratamiento multidisciplinaria que promueve esta iniciativa.

- El desentendimiento de la clase política de ciertas necesidades de servicio público relacionados con la educación, la asistencia sociosanitaria, etc.

- El desmantelamiento de estructuras organizativas estrechamente relacionadas con los propósitos de desarrollo local, como los agentes y las agencias de desarrollo local.

De esta manera, a la nómina de entidades locales que nunca incorporaron en su agenda de trabajo los principios de desarrollo local se han unido ahora el grupo que, habiéndolas adoptado, posiblemente no han sabido valorar las contrastadas bondades y rentas del desarrollo local. Tiene mucho que ver con esta nueva situación la nueva estructura de poder nacida de las últimas elecciones generales, autonómicas y municipales. La constitución de nuevas mayorías absolutas en muchas corporaciones municipales y en otros órganos de decisión ha permitido "legitimar" las medidas adoptadas y estas han servido para justificar ciertas prácticas como:

- No tener que compartir ni consensuar decisiones con las otras formaciones políticas.

- Atender sólo a razones partidistas, legitimando el derecho a decidir en solitario hasta en aquellas cuestiones no contenidas en el programa electoral.

- Negar a los actores locales la posibilidad de poder jugar un papel activo en la gestión y control de actividad municipal y de que puedan proponer iniciativas que ayuden a movilizar recursos, tanto endógenos y como exógenos, en beneficio de la colectividad.

Coincidiendo con la crisis actual, una de las decisiones políticas adoptadas en relación al desarrollo local que mejor coinciden con este planteamiento ha sido no contar con los técnicos dedicados al desarrollo local: los Agentes de Empleo y Desarrollo Local (AEDL) y, en el caso de haber constituido, de las agencias que las integraban y coordinaban.

Como se ha detectado en algunos estudios sobre la implantación de políticas públicas de desarrollo local en algunas Comunidad Autónomas, no cabe duda de que el trabajo desempeñado por los AEDL no ha estado exento de problemas y deficiencias.

En el caso concreto de la Comunidad Valenciana, Calvo (2010) hace referencia a una serie de elementos propios del modelo de desarrollo local que dificultan la obtención de los resultados deseados. Cita como una de las más destacadas del desarrollo el que sea "un modelo de carácter personalista, de- 
rivado principalmente de la excesiva identificación del citado modelo con la figura operativas del mismo, el Agente de Empleo y Desarrollo Local" (p.41). Es esta una consideración central en el trabajo presentado por Calvo sobre la evolución del desarrollo local en España, en el que, además de vislumbrar posibles horizontes en la evolución del modelo, formula la pregunta de "si el modelo de desarrollo local en España podría subsistir sin la existencia de los técnicos AEDL" (p. 41).

Desde nuestro punto de vista, las importantes dificultades económicas a las que nos ha abocado la crisis y, especialmente, el importante número de parados, es una ocasión inmejorable para redefinir el modelo de desarrollo local en España, repensar objetivos y funciones, etc.

En las actuales circunstancias, eliminar los AEDL del organigrama administrativo de los ayuntamientos y otras entidades públicas es un tremendo despropósito que ha generado importantes inconvenientes en la gestión de los recursos locales, al quedar desatendidas una serie de campos estrechamente vinculados a su trabajo. Su desaparición ha perturbado negativamente la continuidad de las políticas activas de empleo y, entre otras cosas, ha propiciado el abandono de una muy rentable práctica mediadora entre los poderes públicos, el mercado y los actores locales.

En realidad, existen tora una serie de aptitudes políticas que han condicionado el normal desarrollo y evolución de las actividades propias del desarrollo local. En el caso valenciano, los argumentos que utiliza Calvo (2010) para demostrar la falta de convencimiento de la mayoría de los dirigentes políticos locales a favor del desarrollo local son los siguientes:

- La escasez de recursos propios de las Corporaciones locales dedicados al desarrollo local como una clara limitación.

- El hecho de que un porcentaje muy alto de técnicos relacionados con el desarrollo local depende de la concesión de subvenciones de diferente procedencia.

- La falta de compromiso con los principios del desarrollo local y de promoción de los técnicos implicados.

- La inexistencia de una estructura interna de funcionamiento eficiente.

- La utilización del AEDL para la realización de actividades no adscritas a su perfil.

Tratado el tema de desarrollo local con la objetividad y profundidad necesarias, las conclusiones a las que llegan diferentes autores y muestran la existencia de procesos similares en otros casos de estudio.

\section{TRabajo, EMPleo, ocupación y desarRollo Local}

Cabría pensar que, al menos en el caso balear, las decisiones adoptadas por los poderes públicos en relación al desarrollo local responde generalizada apreciación de que las acciones desarrolladas no han tenido los resultados 
esperados y de que, en la actualidad, tampoco sirven para luchar contra la profunda crisis que nos atenaza. Sin embargo, ambos argumentos pierden consistencia si se tienen en cuenta que, desde los diferentes ámbitos de decisión del Gobierno Balear que podría tener mejor relación con el desarrollo local, no se ha llevado a cabo ningún estudio que permita confirmar este extremo, valorar de forma concluyente el trabajo desplegado por los AEDL ni evaluar los posibles beneficios alcanzados en los municipios en los que se ha desplegado esta actividad.

Algo tendrá que ver la patética situación a la que se ha llegado en las islas con el tratamiento del tema por pate del Gobierno Balear desde la lógica de su actual estructura competencial ya que no existe ninguna consejería que concluya en su denominación genérica los términos trabajo, ocupación, empleo o similares. En plena crisis, sorprende que en actual organigrama del Gobierno Balear no se haya mantenido y potenciado unidades como la Conselleria de Treball y Formació, establecida en la anterior legislatura.

No deja de sorprender también que, como resultado de la nueva estructura y orientación política del actual gobierno autonómico, el Servei d'Ocupació de les Illes Balears (SOIB) haya cambiado de domicilio: desvinculado de la ya inexistente Conselleria de Treball y Formació, incompresiblemente ha pasado a integrarse en la Conselleria d'Educació, Cultura i Universitats. Tales decisiones hacen pensar que las únicas soluciones propuestas desde las altas instancias del Gobierno Balear para afrontar la actual situación se reducen a mantener las de políticas pasivas de empleo y el programa de actividades formativas para sectores especialmente vulnerables.

En la actualidad, el intento de alcanzar los objetivos de lucha contra el déficit público y de saneamiento de las arcas públicas, basándose tan sólo en el diseño de políticas de ahorro, ha llevado a una preocupante paralización económica y crispación de las clases sociales más desfavorecidas. La drástica reducción de las partidas presupuestarias que antes se dedicaban a la promoción del desarrollo local forma parte de un conjunto de decisiones tan precipitadas como injustificables. En este sentido, posiblemente uno de los mayores disparates cometidos por el Gobierno central ha sido la práctica eliminación de la partida dedicada a la promoción de políticas activas de empleo, decisión que ha venido acompañada por la cancelación de la transferencia de los fondos que el Ministerio de Empleo dedicaba a cofinanciar (el 80\% del coste final) la contratación de los AEDL en las diferentes comunidades autónomas.

Ante esta situación, parece ser que mientras algunos gobiernos autonómicos han debilitado una serie de mecanismo para cubrir el coste correspondiente en la contratación de AEDL, en las Islas Balears se decidió en 2012 suspender el programa, afectando negativamente a 73 trabajadores. De acuerdo con este proceso, los pocos AEDL que en la actualidad siguen prestado su servicio en los municipios insulares son aquellos que, bajo esta denominación u otra simi- 
lar, han accedido a la categoría de funcionarios o aquellos otros que aún siguen beneficiándose de un contrato temporal en fase terminal, a punto de caducar.

Se ha desactivado así un plan estratégico que venía funcionando en las islas desde 2001, año en que se produjo el traspaso de competencias en materia de trabajo, ocupación y formación desde el Instituto Nacional de Empleo (INEM) al Servei d'Ocupació de les Illes Balears (SOIB) del Gobierno Balear. Desde entonces y hasta hace poco, este servicio ha tenido un especial protagonismo en materia de desarrollo local, entre otras cosas por atender los siguientes objetivos:

- La implantación de políticas activas de ocupación, de dinamización económica y laboral en los municipios que lo solicitasen.

- La creación de la red balear de AEDL, la Xarxa d'Agents d'Ocupació i Desenvolupament Local, con la intención de que fuera un espacio de formación, coordinación, comunicación y diálogo de los AEDL de las islas.

- La gestión y control de les subvenciones para la contratación de AEDL.

Abundando algo más en el tema, interesa destaca que en el modelo de trabajo presentado en 2009 por parte de la entonces Conselleria de Treball y Formació del Gobierno Balear, basado en un proyecto de coordinación entre los agentes territoriales (orientadores y AEDL), se contemplaban las siguientes líneas de actuación:

1. Fomento de las políticas de desarrollo local

2. Consolidación de la red de desarrollo local

3. Profesionalización de los AEDL

4. Coordinación de los agentes territoriales

5. Apoyo técnico del SOIB

6. Coordinación con el Observatorio de Trabajo

Los datos sobre la evolución de la dotación económica asignada por el Gobierno balear para sufragar parte de los gastos derivados de la contratación de AEDL en las islas y del número de agentes contratados que se recogen en el Cuadro $\mathrm{n}^{\mathrm{o}} 1$ ayudan a identificar uno de los aspectos que, desde el punto del desarrollo local, mejor pueden identificar el importante cambio de tendencia de nuestros políticas. Aunque no disponemos de más datos que los que aparecen en dicho cuadro, sabemos que el número de AEDL contratados en las Islas Baleares llegó a ser de un centenar, un máximo histórico que posiblemente se alcanzó en 2011.

Cuadro $n^{o}$ 1. Dotación económica destinada a la contratación de AEDL en las Islas Baleares y número de agentes contratados

\begin{tabular}{|c|c|c|}
\hline Año & Dotación económica (euros) & Número de AEDL contratados \\
\hline 2005 & 27.046 & 54 \\
\hline 2010 & 108.182 & 80 \\
\hline 2013 & 0 & 0 \\
\hline
\end{tabular}

Fuente: 
3. LA RESPUESTA DE LA CIUDADANÍA A LA CRISIS POLÍTICA Y ECONÓMICA: DE LA RESISTENCIA A LA RESILIENCIA

En los apartados anteriores hemos intentado prestar atención a dos cuestiones básicas. Por una parte, hemos intentado justificar nuestro posicionamiento a favor de un modelo de desarrollo local basado en la intervención activa y responsable de los diferentes actores sociales, políticos y económicos, desde una perspectiva amplia, integrada y sostenible. Por otra parte, pretendemos dejar constancia de que el cambio de orientación política de los últimos años en esta materia ha generado un cada vez mayor distanciamiento entre la clase política y la ciudadanía en la gestión de los asuntos públicos, desatendiéndose uno de los principios fundamentales del desarrollo local.

Posiblemente ahora más que nunca, la delicada situación a la que se ha llegado exige una decidida actuación para salir del "tremendo pozo de descrédito acumulado" (SOBERATS 2013) en el que han caído (y no parece querer salir) buena parte de nuestros representantes políticos y de sus asesores. El alcance de la crisis, la falta de expectativas que puedan hacer pensar en una pronta corrección de la trayectoria y la indignación popular reclama que se lleven a cabo reformas en profundidad, de carácter estructural.

Precisamente existe un amplio consenso en que una de las asignaturas pendientes de nuestra democracia es la reforma del actual modelo político de convivencia, incapaz de resolver las importantes carencias históricas y atender las crecientes reclamaciones de la sociedad civil.

En algún apartado de este trabajo y en otros (BRUNET 2011) hemos hecho referencia a algunas deficiencias en el desarrollo de la actividad de los AEDL, así como en la incapacidad de la clase política para mejorar la situación general del país. Como ya hemos planteado antes, la difícil situación política, social y económica que atravesamos no puede cambiar aplicando las habituales medidas terapéuticas de carácter epidérmico o estético a las que se nos ha acostumbrado, sino tomar medidas contundentes y sistémicas que permitan regenerar la vida política en nuestro país. Sólo así se podrá tener esperanza de que el cambio alcanzará a todos los componentes del sistema.

Precisamente el funcionamiento de la actividad política y la gestión pública, así como muchas de las decisiones derivadas del desarrollo de esta actividad, permiten detectar una serie de deficiencias que son especialmente visibles sino todo lo contrario: constituyen la verdadera raíz del problema. Las principales carencias de la clase política que nos atrevemos a citar son:

- La falta de formación y capacitación para ejercer actividades políticas.

- La falta de rigor y objetividad en la toma de decisiones.

- La falta de transparencia de las actuaciones desarrolladas: rendición de cuentas. 
- La defensa a ultranza y extemporánea de los principios constitucionales de la democracia representativa a la hora de justificar y legitimar las decisiones.

- La percepción de la puesta en práctica de mecanismos propios de la democracia participativa como una simple deferencia y no como un derecho.

- La infravaloración de las ventajas de dejarse acompañar por la ciudadanía en las tareas de gobierno

- La medida de los problemas y la presentación de soluciones desde una perspectiva exclusivamente partidista.

- La incapacidad para implantar modelos de gestión de calidad y de planificación estratégica.

- La incapacidad por internalizar criterios de sostenibilidad en la gestión política.

Desde nuestra perspectiva (no sabemos si en gran medida condicionada por nuestra percepción especial del proceso de seguido en las Islas Baleares) y a partir de lo expuesto anteriormente, se puede establecer una clara relación entre dos factores: el desinterés por estimular prácticas participativas y políticas de concentración social, y la falta de transparencia de la actividad política. De ahí que otra vez estemos totalmente de acuerdo con SOBERATS (2013) en que "...el descrédito de la política institucional, de la política de los políticos, no procede solo de abusos de poder, de casos de corrupción o de privilegios injustificados. Es también el resultado de la falta de adecuación de la lógica representación-decisión, o de la mecánica Gobierno-oposición", lo que explica que la política sea vista "más como un problema que como parte de la solución".

En realidad, desatender la necesidad de crear espacios de proximidad y de trabajo colaborativo para mejorar la situación de nuestros pueblos y municipios no es otra cosa de rechazar uno de los principios básicos del modelo de desarrollo local: el aprovechamiento eficiente de los recursos endógenos.

Las limitaciones impuestas por la falta de participación de la población local a la hora de diagnosticar problemas y de plantear soluciones, unido al desinterés de los poderes públicos por implementar otros objetivos del modelo de desarrollo local, autoriza a formular la pregunta de si se puede hablar de desarrollo local sin la participación de los poderes públicos. Si bien es cierto que esta pregunta reviste de una mayor complejidad si se plantea desde la perspectiva de lo sostenible (desarrollo local sostenible), es fácil adelantar que no puede existir sostenibilidad si no se cumplen previamente los objetivos del desarrollo local (BRUNET 2011).

Desde una perspectiva tradicional la respuesta tendría que ser negativa si atendemos la consideración explícita que hacen algunos de que uno de sus principales elementos caracterizadores del desarrollo es "su dimensión institucio- 
nal, al estar controlado por la Administración Pública (gobierno central) para asegurar la coordinación de los agentes implicados" (SANCHÍS 1999: 151).

Sin embargo, ante tanto despropósito, no es de extrañar que se haya reducido de forma notable el número de ciudadanos asistentes a actos institucionales como son las sesiones públicas municipales (en muchos casos convocados a deshoras para asegurar la menor asistencia posible de ciudadanos) o su participación en proyectos comunitarios como las Agendas 21 Locales (A21L) u otras iniciativas similares.

La sensación de abandono, orfandad e indignación asumida por un creciente número de personas y colectivos, ha propiciado la adaptación de dos actitudes diferentes, de especial alcance especialmente evidente en tiempos de crisis.

1. La resistencia pasiva

2. La actuación de diferentes mecanismo de resiliencia (entendida como capacidad de las personas o grupos para enfrentarse y sobreponerse a los resultados adversos) y a la implicación en el desarrollo de diferentes iniciativas, generalmente de carácter cooperativo.

Desde la crisis de los 70, pero de manera especial evidente en los últimos años, se ha desatado una auténtica explosión en cadena de nuevas formas de activismo social, en muchos casos gestionados a través de redes sociales. Normalmente aparecen como respuesta a las situaciones de desamparado social, precariedad laboral e instinto de supervivencia de momentos de crisis, aunque la procedencia de sus seguidores de resiliencia activa, cooperativa y responsable revela la adopción de nuevos valores, un cambio cultural en los hábitos de consumo, un nuevo sistema de relaciones personas, así como la adaptación de posiciones críticas frente al consumismo y a favor del desarrollo sostenible.

Se trata de nuevas formas de activismo comunitario que tienen una especial incidencia en el ámbito local y que, en algunos casos, han permitido diversificar las propuestas tradicionales de intervención en materia de desarrollo local y alcanzar los siguientes objetivos:

- Incentivar la movilización ciudadana y la solidaridad

- Priorizar el uso frente a la propiedad de las cosas

- Crear nuevos espacios de trabajo colaborativo

- Favorecer la cohesión social

- Crear mayor proximidad entre la población

- Apostar por fórmulas de consumo sostenibles

- Potenciar la creación de nuevas estructuras organizativas

- Trabajar en red

En el Cuadro ${ }^{\circ} 2$ se identifican una serie de iniciativas concretas, algunas de ellas con un indudable contenido social y que pueden reconocerse como ejemplo de buenas prácticas en el ámbito del desarrollo local sostenible. 
Cuadro $n^{\circ} 2$ Algunos ejemplos de consumo cooperativo

\begin{tabular}{|c|c|c|}
\hline Tipo de iniciativa & $\begin{array}{c}\text { Referencia temporal } \\
\text { Lugar de implantación }\end{array}$ & Características \\
\hline Trueque & Actividad tradicional & $\begin{array}{l}\text { Iniciativas locales son lucrativas que proveen a } \\
\text { la comunidad de información de los productos } \\
\text { y servicios que sus miembros pueden inter- } \\
\text { cambiarse entre sí. } \\
\text { Intercambio de productos y de servicios. En } \\
\text { algunos casos, en las transacciones se utiliza } \\
\text { una moneda propia de valor preestablecido. } \\
\text { Los principios básicos sobre los que se sus- } \\
\text { tenta este mercado son la reciprocidad, la con- } \\
\text { fianza y la ayuda mutua. }\end{array}$ \\
\hline $\begin{array}{l}\text { Time Dollar. Time } \\
\text { Bank.. bancos del } \\
\text { Tiempo (BdT) }\end{array}$ & $\begin{array}{l}\text { Estados Unidos } \\
\text { Años } 80\end{array}$ & $\begin{array}{l}\text { Los Bancos del Tiempo ayudan a resolver } \\
\text { problemas básicos a personas en situación de } \\
\text { precariedad económica, en situación de de- } \\
\text { pendencia o aquellas otras que relaman cierto } \\
\text { tipo de servicio, en la mayoría de los casos no } \\
\text { convencional. } \\
\text { La unidad de cambio suele ser la hora, utili- } \\
\text { zándose en ocasiones monedas sociales. } \\
\text { Las personas implicadas pueden recibir ser- } \\
\text { vicios y satisfacer así ciertas necesidades sin } \\
\text { devengar remuneración económica en alguna } \\
\text { sino con las mismas horas de servicios com- } \\
\text { pensatorios. } \\
\text { Funciona en red y general bajo la supervisión } \\
\text { de un coordinador. En algunos BdT, el coordi- } \\
\text { nador se erige también como dinamizador de } \\
\text { actividades lúdicas, recreativas o de otro tipo. }\end{array}$ \\
\hline $\begin{array}{l}\text { Proyectos de reci- } \\
\text { claje y reutilización: } \\
\text { Millor que Nou: } \\
100 \% \text { Vell }\end{array}$ & Barcelona, 1997 & $\begin{array}{l}\text { Campaña que ofrece diferentes posibilidades } \\
\text { para algar la vida útil de los objetos y reducir } \\
\text { la generación de basura. Para ello se ofrecen } \\
\text { diferentes talleres de reparación, tiendas de } \\
\text { segunda mano y webs de intercambio }\end{array}$ \\
\hline $\begin{array}{l}\text { Proyectos de recicla- } \\
\text { je y reutilización: Re- } \\
\text { parar Millor que Nou }\end{array}$ & & $\begin{array}{l}\text { Proyecto de asesoramiento técnico con el ob- } \\
\text { jetivo de facilitar la autoreparación. }\end{array}$ \\
\hline BookCrossing & 2001 & $\begin{array}{l}\text { Práctica gratuita de dejar libros en lugares pú- } \\
\text { blicos para que los recojan otros lectores, que } \\
\text { después procederán de la misma manera. La } \\
\text { idea es liberar libros "en la jungla" para que } \\
\text { sean encontrados por otras personas }\end{array}$ \\
\hline
\end{tabular}




\begin{tabular}{|c|c|c|}
\hline Tipo de iniciativa & $\begin{array}{l}\text { Referencia temporal } \\
\text { Lugar de implantación }\end{array}$ & Características \\
\hline $\begin{array}{l}\text { Bike Crossing. BKC. } \\
\text { Cruce de bicicletas }\end{array}$ & 2009 & $\begin{array}{l}\text { Práctica de liberar en lugares públicos bicicle- } \\
\text { tas usadas y arregladas para otros usuarios ne- } \\
\text { cesitados las recojan y las usen por un tiempo } \\
\text { indeterminado. La idea es arreglar bicicletas } \\
\text { de cualquier tamaño y edad, que ya no se usen, } \\
\text { y dejarlas para que otras personas las utilicen } \\
\text { de nuevo. }\end{array}$ \\
\hline $\begin{array}{l}\text { Sistemas de bicicle- } \\
\text { tas compartidas }\end{array}$ & Copenhague, 1995 & $\begin{array}{l}\text { Consiste en poner a disposición de los usua- } \\
\text { rios una serie de bicicletas para que sean uti- } \\
\text { lizadas temporalmente como medio de trans- } \\
\text { porte y desistan de utilizar el vehículo privado. }\end{array}$ \\
\hline $\begin{array}{l}\text { Carpool o Carpoo- } \\
\text { ling. Coche compar- } \\
\text { tido }\end{array}$ & & $\begin{array}{l}\text { Práctica que consiste en que diversas personas } \\
\text { compartan coche, tanto para viajes periódicos } \\
\text { como para trayectos puntuales. Se pretende re- } \\
\text { ducir la congestión y contaminación del trán- } \\
\text { sito en las grandes ciudades así como facilitar } \\
\text { los desplazamientos a personas que no dispon- } \\
\text { gan de coche propio. }\end{array}$ \\
\hline $\begin{array}{l}\text { Car Sharing. } \\
\text { Coche compartido en } \\
\text { régimen de alquiler }\end{array}$ & & $\begin{array}{l}\text { Modelo de uso de coches compartidos en régi- } \\
\text { men de alquiler: el usuario alquila el vehículo } \\
\text { por cortos períodos de tiempo, habitualmente } \\
\text { por una hora. } \\
\text { Interesante tanto para aquellas personas no } \\
\text { propietarios de coche que quieren hacer uso } \\
\text { ocasional de uno como para aquellos otros que } \\
\text { deseen utilizar un coche de diferentes presta- } \\
\text { ciones al suyo. }\end{array}$ \\
\hline Taxi Compartio & & $\begin{array}{l}\text { Vehículos de transporte colectivo que realiza } \\
\text { una ruta fija. Habilitado para transportar cierto } \\
\text { número de pasajeros, se cobra una tarifa fija } \\
\text { por persona que puede variar según el horario } \\
\text { y la distancia que se recorra. }\end{array}$ \\
\hline $\begin{array}{l}\text { Coworking. } \\
\text { Cotrabajo }\end{array}$ & & $\begin{array}{l}\text { Forma de trabajo que permite a profesionales } \\
\text { independientes, emprendedores y pymes de } \\
\text { diferentes sectores compartir un mismo espa- } \\
\text { cio de trabajo, tanto físico como virtual. Sirve } \\
\text { para desarrollar diferentes proyectos profesio- } \\
\text { nales de manera independiente así como fo- } \\
\text { mentar proyectos conjuntos. }\end{array}$ \\
\hline
\end{tabular}




\begin{tabular}{|l|l|l|}
\hline \multicolumn{1}{|c|}{ Tipo de iniciativa } & $\begin{array}{c}\text { Referencia temporal } \\
\text { Lugar de implantación }\end{array}$ & \multicolumn{1}{c|}{ Características } \\
\hline $\begin{array}{l}\text { Huertos Urbanos, } \\
\text { minihuertos }\end{array}$ & $\begin{array}{l}\text { Huertos comunitarios pensados para sectores } \\
\text { de la población con bajos recursos y pensio- } \\
\text { nistas. } \\
\text { Acoge diferentes modalidades de agricultura } \\
\text { respetuosas con el medio ambiente. } \\
\text { Se convierten en espacios de relación social y } \\
\text { de intercambio de productos. } \\
\text { En algunos casos se promueven talleres for- } \\
\text { mativos y el desarrollo de actividades lúdicas, } \\
\text { recreativas o de otro tipo. }\end{array}$ \\
\hline Couchsurfing. & & $\begin{array}{l}\text { Sistema de intercambio de alojamientos en ca- } \\
\text { sas de gente no conocida previamente y que } \\
\text { contacta a través del página WEB. Se duerme } \\
\text { en el sofá y se comparte experiencias y cono- } \\
\text { cimientos, se realizan actividades conjuntos, } \\
\text { etc. }\end{array}$ \\
\hline
\end{tabular}

Fuente:

El cuadro anterior contiene una información que, como es nuestra intención, deberá ser trabajado con más rigor. Para caracterizar dichas propuesta, en algunos casos se ha producido de forma literal más o menos litoral el descriptor de algunas WEB.

Algunos de los ejemplos descritos desarrollan la actividad bajo los principios de la economía no monetaria.

Finalmente, interesa destacar que algunos de los ejemplos elegidos, en España se encuentran aún en la etapa de maduración por la que ya han pasado en otros países y que algunas de ellas han conseguido ser viables económicamente.

\section{CONCLUSIONES}

Este trabajo ha sido realizado desde el estado de indignación producido por los acontecimientos políticos que se van sucediendo en nuestro país, por el deficiente manejo de la crisis y por la incapacidad de los poderes públicos para promover cambios sustanciales del sistema político que permiten mejorar la "insuficiente democracia que existe en España" (NAVARRO, TORRES Y GARZÓN, 2001: 205).

Se plantea la necesidad de acometer el análisis y valoración del desarrollo local a lo largo del proceso y no sólo a partir de un análisis de los resultados obtenidos, adoptando una terminología (calidad total) y una metodología de trabajo parecidas a las utilizadas en las auditorias de gestión empresarial. 
BIBLIOGRAFÍA

ALIÓ I TORRES, M.A, eitora: Ciutadania i Recerca a la Universitat. Esperienciesde col.laboració entre la Ciutadania i recerca Universitaria. Grup de Geógrafs d'Ecologoia Social/2GES. Barcelona.

BELTRÁN, E (2009): Derechos torcidos. Tópicos, medias verdades y mentiras sobre pobreza, política y derechos humanos. Debate. Barcelona.

BRUNET ESTARELLES, P.J. (2006): Estrategias de cooperación y desarrollo territorial sostenible en las Islas Balears. El papel del gobierno regional e insular en la implantación de las Agendas Locales 21 en las Illes Balears. Revista Desarrollo Territorial, 1:375-398. Publicaciones de la Universitat de València.

BRUNET ESTARELLAS, P.J. (2011): L'Agenda 21 i el seu entorn: actors i bones practiques. Estudis OPL n ${ }^{\circ}$ 10. Observatori de Polítiques Locals/ departamento de Cooperació Local. Consell de Mallorca. Palma.

BRUNET ESTARELLAS, P.J. (2012): El control del proceso de implantación y gestión de la A21L. El decreto 123/2002 de implantación de la A21L en los municipios de las Islas Baleares. XI Congreso nacional de Medio Ambiente (CONAMA), Madrid.

BRUNET ESTARELLAS, P.J. AMEIDA GARCÍA, F. y COLL LÓPEZ, M. (2005): Agenda 21, subsidiariedad y cooperación a favor del desarrollo territorial sostenible. Boletín de la Asociación de Geógrafos Españoles $(A G E) \mathrm{n}^{\circ}$ 39: 423-446.

CABRERO, E. (2005): Acción pública y desarrollo local. México, Fondo de Cultura económica.

CALVO PALOMARES, R. (2010): 25 Años de desarrollo local en España: Un gigante con pies de barro. Sociedad y Utopía. Revista de Ciencias Sociales, n 36 ; 39-66, noviembre de 2010.

GONZÁLEZ CANCELA, I. (2011): Guia per incorporar criteris ambientals a la contractació pública. Estudis OPL n 10 . Observatori de Polítiques Locals/ Departament de Cooperació Local. Consell de Mallorca. Palma.

GRAGERA DE LEÓN, F.: La cara y cruz del boniato. El País, 31 de marzo de 2013.

HARVEY, D. (2003): Espacios de Esperanza. Ediciones Akal. Madrid.

KLEIN, J.L. (2006): Geografía y desarrollo local. En: D. Hiernaux/ A. Lindón, dirs. (2006): Tratado de Geografía Humana. Anthropos Editorial/UAM. Barcelona.

KRUGMAN, P. (1912): ¡Acabad ya con esta crisis! Crítica. Barcelona.

MARTÍNEZ PUCHE, A. et al (2008): Sostenibilidad en lso espacios rurales. Proyectos europeos, herramientas participativas, experiencias municipales $y$ territoriales en España. CEDER-AITANA.

MORENO, S.: Aguantar o resistir? Ara, 14 de mayo de 2013: 24. 
NAVARRO, V., TORRES, J. Y CARGÓN, A. (2011): Hay alternativas. Propuestas para crear empleo y bienestar social en España. Sequitur/ATTAC España. Madrid.

NOLLE-NEUMANN, E. (2010): La espiral del silencio. Opinión pública: nuestra piel social. Paidos. Barcelona.

OPL (2009): Qualitat de gestió a l'Administració local. El canvi necessaria en les cultures corporatives. Estudis OPL $\mathrm{n}^{\circ}$ 7, Observatori de Polítiques Locals/ Departament de Cooperació Local. Consell de Mallorca. Palma.

SANCHIS PALACIO, J.R. (1999): Las estrategias de desarrollo local: aproximación medodológica desde una perspectiva socio-económica e integral. Dirección y organización: Revista de dirección, organización y administración de empresas $n^{\circ}$ 21: 147-160.

SOLER, J.: El reverso de la crisis. El País, 12 de mayo de 20113: 41.

STINGLITZ, J.E. (2012): El precio de la desigualdad. Taurus. Madrid.

SUBIRARTS, J.: Apuntes de nueva política. El País, 10 de marzo de 2013.

VALDIVIELSO, J. (2004): Les dimensions socials de la crisi ecológica. Edicions UIB. Palma. 